\title{
Field Testing of Ovicidal-Larvicidal Trap System with Pelletized Extracts of Piper nigrum L. for Aedes Mosquito in Quezon City and Marikina City
}

\author{
Annabelle V. Briones, Alicia G. Garbo, Edmar P. Casa, Hermelina H. Bion, \\ Nuna E. Almanzor and Severino T. Bernardo \\ Industrial Technology Development Institute \\ Department of Science and Technology \\ DOST Complex, Gen. Santos Ave., Bicutan, Taguig City, Philippines
}

\begin{abstract}
Objective. This study was conducted to validate the effectiveness of the ovicidal-larvicidal (OL) trap with the pelletized extracts of Piper nigrum L. in attracting female Aedes aegypti mosquitoes for oviposition and in reducing the number of larvae hatched, thus preventing the emergence of adult mosquitoes.

Methods. The OL trap system containing the developed plantbased pellets from Piper nigrum L. was field tested in Marikina and Quezon City. Paired OL traps were installed outdoors in 150 selected houses in each city. The OL trap is composed of a black painted can, a small strip of lawanit (paddle) for oviposition and extracts of Piper nigrum L. as the ovicide-larvicide solution or plain water. Pelletized form of Piper nigrum L. was prepared by grinding the seeds then mixing it with $1 \%$ previously cooked starch solution at a ratio of 1:1 followed by extrusion and forming into pellets using a granulator then drying. The number of positive OL traps was recorded. Larval mortality and the Ovitrap Index were also determined.
\end{abstract}

Results. Four collections were made using the pelletized form in which 2,314 OL traps were collected. The OL traps with the pelletized form showed a $53.2 \%$ attraction which is significantly higher than that with water ( $p$-value $<0.0001)$. The percent egg and larval mortality in both cities was recorded at $86.2 \%$ while adult emergence is $13.8 \%$. The Ovitrap Index in Marikina was in the range of $53 \%$ to $68 \%$ while OL traps with water have an Ovitrap Index range of 39\% to $65 \%$. In Quezon City, OL traps with the pelletized admixture have an Ovitrap Index range of $35 \%$ to $50 \%$ while those with water have a range of $31 \%$ to $36 \%$.

Conclusion. The overall results indicate that the developed $\mathrm{OL}$ trap system with Piper nigrum $L$. is effective in attracting Aedes aegypti mosquitoes. The $\mathrm{OL}$ traps with extracts of Piper nigrum $L$. were also found to be lethal to larvae.

Key Words: Piper nigrum L., ovicidal-Iarvicidal trap, Iarvicide, ovicide

Corresponding author: Annabelle V. Briones Industrial Technology Development Institute DOST Complex, Gen. Santos Avenue, Bicutan, Taguig City

Telephone: +632 8372071 local 2216

E-mail: avbriones2003@yahoo.com

\section{Introduction}

Several control methods were already developed for the Aedes aegypti mosquito, which include adulticiding and larviciding using insecticides or breeding sources reduction; however, the use of insecticides in the long term should be discouraged because of environmental contamination and the possibility of the insect's development of resistance to insecticides. Aedes aegypti is the primary vector of the dengue virus which infects hundreds of people every year causing dengue fever and the potentially fatal dengue hemorrhagic fever (DHF). In a study on vector indicators for dengue, it was discussed that low human immunity, high mobility of the human population and high transmission rate of the Dengue virus through vector mosquitoes Aedes aegypti and Aedes albopictus are mostly the reasons why Dengue is one of the major medical and health problems of the government. ${ }^{1}$

An OL trap is a device used to control the Aedes mosquito population. It can monitor, control and detect Aedes mosquito population which preempts any impending dengue outbreaks. Aside from being used in Aedes aegypti surveillance, an OL trap can be lethal to eggs once an ovicide-larvicide is incorporated on the oviposition substrate. OL traps allow oviposition but prevents adult emergence thus reduces the Aedes mosquito densities.

A study on the in vitro activity of the crude extracts of dried whole black pepper seed (Piper nigrum L.) on Aedes aegypti larvae revealed that it is a potential natural insecticide against mosquitoes in larval stages. ${ }^{2}$ Another study on the efficacy of some botanical extracts against medically important mosquitoes in the Philippines found that Piper nigrum L. prepared as paminta treated sawdust exhibits larvicidal property. ${ }^{3}$ These studies show its ability to kill the larvae as soon as they hatch from the egg stage, thus regulating the population of larvae resulting to depletion of immature mosquitoes.

An OL mosquito trap, especially when ovicide-larvicide solutions are added, offers a simple, cheap and efficient tool for surveillance and control of mosquito. The primary objective of this project is to field test the developed ovicidallarvicidal admixture of Piper nigrum L. for Aedes aegypti in Quezon City and Marikina City. 


\section{Methods}

Black Piper nigrum L. seeds were procured in Batangas, Philippines for this study. Pelletized form of Piper nigrum L. will be added to the OL trap to function as the ovicidelarvicide solution.

\section{Preparation of the pelletized extract of Piper nigrum L.}

The Piper nigrum L. admixture in pellet form was prepared by mixing the ground seeds with $1 \%$ previously cooked starch solution at a ratio of 1:1. The mixture was then extruded and formed into pellets using a granulator, followed by drying.

\section{Distribution of kits to $\mathbf{1 5 0}$ households per sampling site}

Quezon City and Marikina City, among other cities in Metro Manila, were chosen because of the high incidence of dengue in 2009. In each city, the barangay which has the recorded highest incidence of dengue was selected as the site of the study. With a designated starting point, 150 adjacent houses who agreed to participate were installed with OL traps. There is one operation officer and 20 sample managers employed in each city to monitor the OL traps.

The kit is comprised of 2 sets of black painted can, ovipaddles and ovicidal-larvicidal pellets for test sample and plain water for negative control. In each house, the kit was placed outdoors, one foot apart in a similarly low, dark and protected place outside the house. OL traps were collected and replaced immediately with the ovicidallarvicidal pellets and ovipaddles every sixth day in a week for one month. The operation officer gathered all the samples and submitted it to ITDI for reading and analysis.

\section{Data processing}

For each day of collected OL traps, the number of eggs and larvae were counted and recorded in the ITDI laboratory. Data was entered in a database prepared in an MS Excel spreadsheet by a data encoder. Data was checked for accuracy and completeness by another member of the team. The MS Excel spreadsheet was then converted to STATA Ver. $10.1^{3}$ for data analysis.

\section{Analysis of data}

Descriptive statistics, such as mean and standard deviation of the number of eggs and larvae hatched across all houses, were generated. These statistics were calculated by trial. Significant differences in the mean number of eggs and larvae hatched were assessed using paired t-tests at each time point. The $5 \%$ level of significance for the statistical tests was applied. The percentage of OL traps positive for Aedes aegypti eggs and live larvae across all houses was also determined for each type of OL trap. McNemar's test ${ }^{4}$ was used to determine significant differences in the occurrence of eggs and larvae in the OL traps. The percentage of OL traps with eggs and mean number of eggs indicated the attractiveness of the OL trap for oviposition. The percentage of OL traps with live larvae and mean number of live larvae measured the ovicidal-larvicidal property of the OL traps. Statistical analyses for this study were generated using STATA Ver 10.1. ${ }^{5}$

The Ovitrap Index is a measurement of mosquito eggs in specified geographic locations which, in turn, reflects the distribution of Aedes mosquitoes. It is determined using the formula:

Ovitrap Index $(\%)=\underline{\text { Number of positive traps with test solution }} \times 100$ Number of traps installed and collected

\section{Limitations of the study}

The use of OL traps as monitoring devices to predict a dengue outbreak was not considered in the study design while the parameters for effective OL trap system was based on its attractancy and ovitrap index. The study is also limited only to field testing of the OL traps with pepper (Piper nigrum L.) based ovicide-larvicide solutions. Water was used as the negative control for comparison with the test solutions. Exposure of the OL traps with water solution also followed the same exposure time with the test solution which is 6 days for comparison. Statistical analysis was based on McNemar's test. ${ }^{4}$

\section{Results}

\section{A. Piper nigrum L. pellets}

A total of 2314 OL traps were collected in a period of 4 weeks, half of which contained the Piper nigrum L. pellets while the other half contained plain water. Figure 1 shows that in the first collection, $43.9 \%$ of the OL traps with pelletized pepper and $37.1 \%$ of OL traps with plain water were found positive with eggs/larvae and this difference was statistically significant $(\mathrm{p}=0.0370)$. In the second collection, $57.4 \%$ and $53.6 \%$ of the OL traps with pelletized pepper and plain water were found positive with eggs/larvae, respectively. This is again statistically significantly different $(p=0.0010)$. In the third collection, $51.5 \%$ of OL traps that were positive with eggs/larvae in the test solution was significantly greater than the $36.6 \%$ in the control solution $(p<0.0001)$. In the fourth collection, $59.4 \%$ of OL traps with pelletized pepper and $50.9 \%$ of OL traps with plain water were found positive with eggs/larvae. Again, the difference was found to be statistically significant $(\mathrm{p}=0.0080)$. Overall, $53.2 \%$ of the OL traps with the pelletized form are positive with eggs/larvae while $42.8 \%$ of those with water have eggs/larvae. This difference was found to be statistically significant ( $p$-value $<0.0001)$.

\section{B. Larval Mortality}

The egg and larval mortality in both cities were also determined (Table 1). In Quezon City, the OL traps with the ovicidal-larvicidal pellets were lethal to $86.2 \%( \pm 2.6 \mathrm{SD})$ of the eggs or larvae. Correspondingly, $13.8 \%$ ( $\pm 2.6 \mathrm{SD})$ of 
Testing of Ovicidal-Larvicidal Trap System for Aedes Mosquito with Pelletized Extracts of Piper nigrum L.

these were able to emerge into adults. Similarly in Marikina City, the \% egg and larvae mortality is $86.2 \% \pm 3.0 \mathrm{SD}$ while $\%$ adult emergence is $13.8 \% \pm 3.07 \mathrm{SD}$.

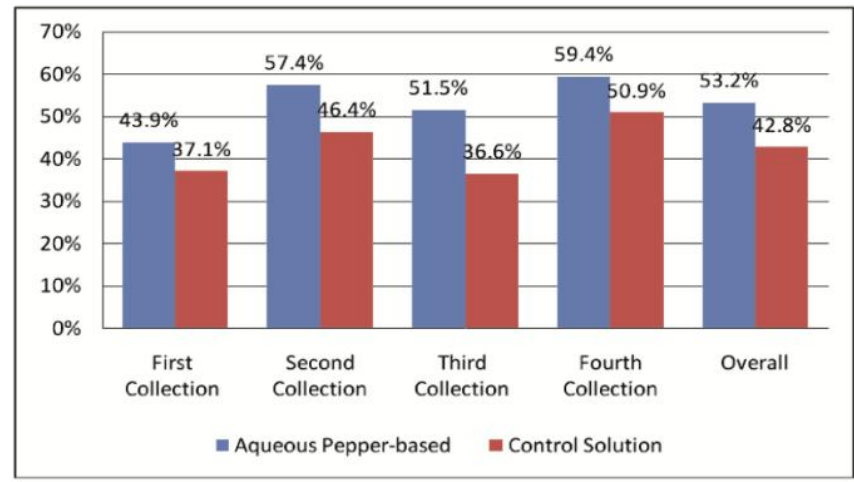

Figure 1. Distribution of households with positive OL traps using the pelletized pepper test solution and control.

Table 1. Percent egg and larval mortality and percent emergence of adult mosquito using pelletized pepper

\begin{tabular}{ccccc}
\hline \multirow{2}{*}{ Week } & \multicolumn{2}{c}{ Quezon City } & \multicolumn{2}{c}{ Marikina City } \\
\cline { 2 - 5 } & $\begin{array}{c}\text { \% Egg } \\
\text { \&Larvae } \\
\text { Mortality** }\end{array}$ & $\begin{array}{c}\text { \% Adult } \\
\text { Emergence* }\end{array}$ & $\begin{array}{c}\text { \% Egg \& } \\
\text { Larvae } \\
\text { Mortality** }\end{array}$ & $\begin{array}{c}\text { \% Adult } \\
\text { Emergence* }\end{array}$ \\
\hline $\mathbf{1}$ & 89.4 & 10.6 & 84.6 & 15.4 \\
$\mathbf{2}$ & 84.8 & 15.2 & 88.2 & 11.8 \\
$\mathbf{3}$ & 87.1 & 12.9 & 82.8 & 17.2 \\
$\mathbf{4}$ & 83.6 & 16.4 & 89.2 & 10.6 \\
Average & $86.2 \pm 2.6$ & $13.8 \pm 2.6$ & $86.2 \pm 3.0$ & $13.8 \pm 3.07$ \\
\hline *observed no. of adult emergence/total no. of observed egg and larvae x $100 \%$ \\
***termined by subtracting \% emergence from 100
\end{tabular}

\section{Ovitrap Index $(\%)$ per area}

The ovitrap index of the pelletized admixture is presented in Table 2. The positive ovitrap index for Marikina was in the range of $53 \%$ to $68 \%$ as compared to the negative control which was about 39\% to 65\%. In Quezon City, the positive ovitrap index showed a range of $35 \%$ to $50 \%$ as compared to the negative control which was about $31 \%$ to $36 \%$.

Table 2. Ovitrap index of OL traps with larvicidal pellet and water

\begin{tabular}{ccccc}
\hline & \multicolumn{4}{c}{ Ovitrap Index (\%) } \\
\cline { 2 - 5 } & WK1 & WK2 & WK3 & WK4 \\
\hline Marikina City & 53.3 & 64.0 & 59.3 & 68.0 \\
Test Solution & $(\mathrm{p}=0.0231)$ & $(\mathrm{p}=0.1573)$ & $\begin{array}{c}\mathrm{p}=0.0001) \\
(\mathrm{p}=0.4349)\end{array}$ \\
Control (Water) & 43.1 & 57.3 & 39.3 & 64.7 \\
$(\mathrm{p}=0.0231)$ & $(\mathrm{p}=0.1573)$ & $(\mathrm{p}=0.0001)$ & $(\mathrm{p}=0.4349)$ \\
Quezon City & & & & \\
Test Solution & 34.8 & 50.4 & 43.4 & 50.3 \\
& $(\mathrm{p}=0.4561)$ & $(\mathrm{p}=0.0009)$ & $(\mathrm{p}=0.0231)$ & $(\mathrm{p}=0.0039)$ \\
Control (Water $)$ & 31.2 & 34.8 & 33.8 & 36.4 \\
& $(\mathrm{p}=0.4561)$ & $(\mathrm{p}=0.0009)$ & $(\mathrm{p}=0.0231)$ & $(\mathrm{p}=0.0039)$ \\
\hline
\end{tabular}

\section{Discussion}

As demonstrated in several studies ${ }^{5,6,7}$ on Aedes aegypti mosquitoes, it is generally accepted that these mosquitoes do not just breed in clean water. This study further showed that the Aedes aegypti mosquitoes deposited more eggs more frequently in OL traps with extracts of Piper nigrum L. Another study by the author herself on oviposition behavior effects of the pelletized admixture of Piper nigrum L. on Aedes aegypti mosquitoes reveals that the solution exhibited an increasing rate of oviposition attraction of female Aedes aegypti to increasing rate of concentration with an average of $70 \%$ attraction at $1000 \mathrm{ppm}$ as compared to $30 \%$ attraction to OL traps with water alone. In that same study, it was also established that Piper nigurm L. extracts can also be used to control larval instars as exhibited in the larvicidal bioassay.

Results in this study showed that there was a statistically significant difference in the positivity and mean number of eggs between OL traps with extracts of Piper nigrum L. and OL traps with water. Moreover, larval mortality is high when extracts of Piper nigrum $L$. were added, thus establishing that OL traps, when infused with extracts of Piper nigrum L., indeed becomes more effective in attracting Aedes mosquitoes and at the same time becomes more lethal to hatched larvae than OL traps with plain water. The OL traps are effective, safe, economical and environmentally safe. Control programs can be done using the OL trap infused Piper nigrum $L$. to decrease the population of Aedes mosquitoes and eventually reduce the incidence of Dengue.

\section{Acknowledgment}

Sincerest thanks to Congressman Feliciano Belmonte, Mayor Herbert Baustista and Vice-Mayor Joy Belmonte of Quezon City, Congressman Miro Quimbo and Mayor Marides Fernando of Marikina City. Special thanks to Bgy. Captain Lito Bernardino and Debbie Cabanag of Bagbag, Quezon City.

\section{References}

1. delas Llagas LA. Establishment of sensitive vector indicator for Dengue Surveillance, DOH Research Compendium 1993-2001.

2. Patricio MGDC, Bravo MVA, Sabit MR, Librojo-Basilio NT. In vitro activity of the crude extracts of dried black pepper (Piper nigrum Linnaeus, 1753) whole fruits on Aedes (Stegomyia) aegypti (Linnaeus, 1762) Larvae Philipp J Vet Med. 2006; 43(2):83-8

3. Cruz IRF, delas Lllagas LA. Efficacy of some Philippine botanical extracts against medically-important mosquitoes. UPM Journal. 1996; 2(2):11.

4. McNemar Q. Note on the sampling error of the difference between correlated proportions or percentages. Psychometrika. 1947; 12(2):153-7.

3. StataCorp. Stata Statistical Software: Release 10. College Station, TX: StataCorp LP. 2007.

5. Nazni WA, Lee HL, Wan Rozita WM, et.al., Oviposition behaviour of Aedes albopictus in Temephos and Bacillus thuringiensis israelensis-treated ovitraps. Dengue Bulletin. 2009; 33:209-17.

6. Lourenço-de-Oliveira R, Lima JB, Peres R, Alves Fda C, Eiras AE, Codeço CT. Comparison of different uses of adult traps and ovitraps for assessing dengue vector infestation in endemic areas. J Am Mosq Control Assoc. 2008; 24(3):38792.

7. Santos SRA, Melo-Santos MAV, Regis L, Albuquerque CMR. Field evaluation of ovitraps consociated with grass infusion and Bacillus thuringiensis var. israelensis to determine oviposition rates of Aedes aegypti. Dengue Bulletin $2003 ; 27: 156-62$. 